\title{
Transcription factor signal transducer and activator of transcription 6 (STAT6) is an inhibitory factor for adult myogenesis
}

\author{
Mitsutoshi Kurosaka ${ }^{1}$, Yuji Ogura ${ }^{1 *}$ (D), Shuichi Sato ${ }^{2,3}$, Kazuhisa Kohda $^{1}$ and Toshiya Funabashi ${ }^{1}$
}

\begin{abstract}
Background: The signal transducer and activator of transcription 6 (STAT6) transcription factor plays a vitally important role in immune cells, where it is activated mainly by interleukin-4 (IL-4). Because IL-4 is an essential cytokine for myotube formation, STAT6 might also be involved in myogenesis as part of IL-4 signaling. This study was conducted to elucidate the role of STAT6 in adult myogenesis in vitro and in vivo.

Methods: Myoblasts were isolated from male mice and were differentiated on a culture dish to evaluate the change in STAT6 during myotube formation. Then, the effects of STAT6 overexpression and inhibition on proliferation, differentiation, and fusion in those cells were studied. Additionally, to elucidate the myogenic role of STAT6 in vivo, muscle regeneration after injury was evaluated in STAT6 knockout mice.

Results: IL-4 can increase STAT6 phosphorylation, but STAT6 phosphorylation decreased during myotube formation in culture. STAT6 overexpression decreased, but STAT6 knockdown increased the differentiation index and the fusion index. Results indicate that STAT6 inhibited myogenin protein expression. Results of in vivo experiments show that STAT6 knockout mice exhibited better regeneration than wild-type mice 5 days after cardiotoxin-induced injury. It is particularly interesting that results obtained using cells from STAT6 knockout mice suggest that this STAT6 inhibitory action for myogenesis was not mediated by IL-4 but might instead be associated with p38 mitogenactivated protein kinase phosphorylation. However, STAT6 was not involved in the proliferation of myogenic cells in vitro and in vivo.
\end{abstract}

Conclusion: Results suggest that STAT6 functions as an inhibitor of adult myogenesis. Moreover, results suggest that the IL-4-STAT6 signaling axis is unlikely to be responsible for myotube formation.

Keywords: Myotube, Myoblast fusion, Differentiation, Primary myoblast, Interleukin-4

\section{Background}

The skeletal muscle, which constitutes $40 \%$ of human body mass, is indispensable for locomotion, respiration, and metabolism [1,2]. The skeletal muscle comprises numerous myofibers, each of which contains multiple postmitotic myonuclei. During the formation of multinucleated

\footnotetext{
*Correspondence: yuji_ogura@marianna-u.ac.jp

'Department of Physiology, St. Marianna University School of Medicine, Kawasaki, Kanagawa 216-8511, Japan

Full list of author information is available at the end of the article
}

myofibers in adults, resident myogenic stem cells undergo a unique process called myogenesis [3-5]. Upon request, myogenic stem cells are activated and committed to differentiation. The activated myogenic stem cells (i.e., myoblasts) subsequently fuse together or with (nascent) myotubes to form mature myofibers [3, 6-9]. Obstruction of myogenesis inhibits proper muscle regeneration after injury, leading to decline of skeletal muscle function [3-5]. Many molecules have been presently identified as triggering and coordinating myogenic differentiation, fusion, and 
maturation of myofibers $[8,10,11]$. Nevertheless, the mechanisms have not been fully elucidated.

The signal transducer and activator of transcription 6 (STAT6) plays a fundamentally important role in immune cells' cellular function $[12,13]$. For instance, STAT6 is involved in $\mathrm{T}$ cell proliferation [14]. Also, its activation prevents apoptotic cell death in B cells [15]. It is also involved in the fusion of macrophages to generate multinucleated giant cells in response to inflammation [16]. Recent studies have also demonstrated the involvement of STAT6 in microglial activation in the brain tissue [17]. Nevertheless, the role of STAT6 in peripheral tissues remains unclear.

Earlier studies have revealed STAT6 as an important target of interleukin (IL)-4 in nonmuscle cells [12, 13, 17]. After IL-4 stimulation, STAT6 gets activated by phosphorylation and functions as a transcription factor to promote context-dependent gene expression [12, 13, 17]. In this regard, IL-4 is an essential molecule for myogenesis. Studies have demonstrated that the nuclear factor of activated $\mathrm{T}$ cells 2 (NFATc2), a calcium-sensitive transcription factor, specifically localizes nascent myotubes and stimulates IL-4 secretion during myoblast differentiation [18-21]. The secreted IL-4 binds to IL-4 receptor alpha (IL-4R $\alpha$ ) on the surrounding myoblasts to promote the fusion of those myoblasts with nascent myotubes [21]. These results suggest that STAT6 is also involved in myotube formation under the control of IL-4. Nevertheless, the function of STAT6 at any stage of myogenesis remains unknown. Therefore, this study was designed to elucidate whether STAT6 can be implicated in adult myogenesis in vitro and in vivo.

\section{Methods}

\section{Animals}

Wild-type (WT) male C57BL/6J mice were purchased from SLC Inc. (Hamamatsu, Shizuoka, Japan). R26-CAG-LoxPmonomeric teal fluorescent protein 1 (mTFP1) mice (B6; 129S6-Gt (ROSA)26Sor ${ }^{\text {tm1.1(CAG-mTFP1)Imayo }}$ ) were obtained from RIKEN (ID: RBRC05147; Tsukuba, Ibaraki, Japan)

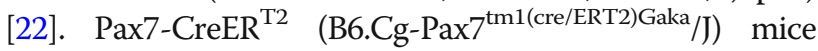
were purchased from The Jackson Laboratory (ID: 017763; ME, USA) [23]. Tamoxifen-inducible muscle stem cellspecific mTFP1-expressing mice were generated by crossing R26-CAG-LoxP-mTFP1 and Pax7-CreER ${ }^{\mathrm{T} 2}$ mice. STAT6-knockout (KO) mice (B6;129P2-Stat6<tm1Aki>/ AkiRbrc) were obtained from RIKEN (ID: RBRC00958) [13]. Animals were maintained in an animal facility $\left(25^{\circ} \mathrm{C}\right.$, $55 \%$ relative humidity, lights on $0600-1800 \mathrm{~h}$ ). All animals had a BL/6 genetic background and were used at 7-8 weeks of age.

\section{Cell culture}

Primary myoblasts were isolated from the hind limb muscles of WT, STAT6-KO, and muscle satellite cell- specific mTFP1-expressing mice as described in earlier reports of the literature $[24,25]$. The expression of mTFP1 was induced by tamoxifen injection $(2 \mathrm{mg} / \mathrm{animal}$, four consecutive days) at 1 week before isolation. After purification by preplating, myoblasts were maintained in growth medium (GM, 20\% fetal bovine serum, 1\% penicillin/ streptomycin, and Ham's F-10; Life Technologies Inc., CA, USA). The medium was supplemented with $5 \mathrm{ng} / \mathrm{mL}$ basic fibroblast growth factor (Peprotech Inc., NJ, USA). When cells attained approximately $90 \%$ cellular confluency, fusion of the myoblasts was induced by switching from GM to differentiation medium (DM, 2\% house serum, $1 \%$ penicillin/streptomycin, and Dulbecco's modified Eagle's medium; Life Technologies Inc.).

\section{Adenovirus construction and infection}

Adenoviruses carrying mouse STAT6 were generated (AdEasy Adenoviral Vector System; Agilent Technologies Inc., CA, USA) as described in the literature [25]. Mouse STAT6 was amplified from mouse cDNA and ligated into the vector (RedTrack-CMV; Addgene, MA, USA) using the KpnI and XbaI sites. The resulting AdTrack-CMVSTAT6 plasmid was linearized with PmeI; then, it was cotransformed into Escherichia coli BJ5183 cells with the pAdEasy-1 plasmid. Clones undergoing AdTrack-Adeasy recombination were selected with kanamycin and were confirmed by enzyme digestion. The recombinant plasmid was linearized with PacI and was transfected into the Adeno-X cell line (Clontech, Manassas, VA, USA) using Lipofectamine 2000 Transfection Reagent (Life Technologies Inc.) packaging into active virus particles. The produced viruses (adenoviral-RFP-STAT6: Ad-STAT6 or adenoviral-RFP-empty: Ad-Ctrl) were amplified further by serial passage to concentrate. At $70 \%$ confluence, Adeno$\mathrm{X}$ cells were infected with the virus and were maintained for 72-96 h. The viral titer was found using an RFPpositive cell number per field. The number of infectious units per milliliter for each well were calculated as (infected cells/field $) \times($ fields/well)/virus volume $(\mathrm{mL}) \times$ dilution factor. For adenovirus infection, myoblasts $\left(1 \times 10^{6}\right)$ were plated and then infected with either a STAT6 vector (Ad-STAT6) or an empty vector (Ad-Ctrl) using the same concentration of infectious units for $6 \mathrm{~h}$. After the infection period, the infected myoblasts were washed carefully and were then maintained in GM for $48 \mathrm{~h}$. Our adenoviral infection had infection efficiency of nearly $100 \%$.

\section{Short hairpin RNA (shRNA)}

Procedures using shRNA were conducted as described in an earlier report [25]. The pLKO.1-mCherry-puro plasmid was provided by Dr. Renzhi Han (The Ohio State University Wexner Medical Center, OH, USA). Target siRNA sequences for mouse STAT6 (GGTTCAGATGCTTTCTGT TAC) were designed using BLOCK-iT RNAi Designer (Life 
Technologies Inc.). After the synthesized siRNA oligonucleotides (Integrated DNA Technologies, Inc., Coralville, IA, USA) were annealed, they were inserted into the plasmid using AgeI and EcoRI sites. The appropriate plasmid was amplified using a standard bacterial culture. Then, the siRNA sequence was validated for the knockdown of STAT6 mRNA in preliminary experiments. Control cells were transfected with the backbone plasmid harboring the scramble sequence (CCTAAGGTTAAGTCGCCCTCG).

\section{Plasmid delivery to myoblasts}

Plasmid DNA was transfected by electroporation (1500 $\mathrm{V}, 10 \mathrm{~ms}$, three pulses) using a transfection system (Neon; Life Technologies Inc.) as described for an earlier study [26]. Our electroporation procedure routinely achieved $70-80 \%$ transfection efficiency at $24 \mathrm{~h}$ posttransfection [26].

\section{IL-4 treatment}

Recombinant IL-4 (R\&D Systems, Minneapolis, MN, USA) was prepared and used similarly to our earlier study [24]. After IL-4 (10 ng/mL) was added to the culture medium in WT and STAT6-KO cells, it was incubated for $48 \mathrm{~h}$. Saline was used as the vehicle.

\section{Evaluation of fusion of myoblasts to nascent myotubes in a cell mixing experiment}

For the cell mixing experiment, we used adenoviral infection to achieve the highest degree of efficiency. The myoblasts isolated from muscle satellite cell-specific $\mathrm{mTFP}^{+}$and WT mice were grown in GM. Based on procedures used for an earlier study [21], mTFP1 $1^{+}$myoblasts were seeded in 24-well plates $\left(0.25 \times 10^{5}\right.$ cells per well) in DM for $48 \mathrm{~h}$ to induce myotube formation and to allow estimation of myotube-myoblast fusion. The original protocol used $24 \mathrm{~h}$ for pre-DM incubation [21], but $48 \mathrm{~h}$ was found to be necessary to form visible myotubes under our experimental conditions. Simultaneously, WT myoblasts were infected with either AdSTAT6 or Ad-Ctrl in GM for $6 \mathrm{~h}$. After the cells were washed, the well was replenished with fresh GM. The cells were then maintained until the following day. After forming mTFP1 $1^{+}$myotubes, Ad-STAT6 or Ad-Ctrl RFP ${ }^{+}$ myoblasts were transferred into the plate to fuse the infected myoblast to mTFP $^{+}$myotubes $\left(0.5 \times 10^{5}\right.$ cells per well). They were maintained for a further $48 \mathrm{~h}$. Then, the fusion index and the number of unfused cells were studied by immunocytochemistry.

\section{Immunocytochemistry}

Cells were prepared for immunocytochemistry as described earlier [24]. Primary antibodies specific to the myosin heavy chain (MyHC, MF-20, 1:50, DSHB) and secondary antibodies conjugated with Alexa Fluor 488
(Life Technologies Inc.) were used for staining. Myonuclei were stained with DAPI. Images of stained cells were captured using a microscope (BZ-9000; Keyence Co.) and were analyzed using Fiji software [27]. The fusion index was defined as the ratio of the number of nuclei in myotubes to the number of nuclei in each image. Myonucleus numbers of the myosin ${ }^{+}$cells were also found. For each experiment, three randomly captured images were analyzed per sample.

\section{Muscle injury}

Into the tibialis anterior (TA) muscle of the left leg of each mouse, $100 \mu \mathrm{l}$ of $10 \mu \mathrm{M}$ cardiotoxin (Latoxan, Valence, France) dissolved in saline was injected [24]. Saline was injected into the TA muscle of the right leg as a vehicle. At 5 days post-injection, both TA muscles were excised for analyses.

\section{Morphological analysis}

Frozen TA muscles were kept below $-20^{\circ} \mathrm{C}$ and were cut using a cryostat (Leica Microsystems $\mathrm{GmbH}$, Wetzler, Germany). Sections were stained with hematoxylin and eosin. Images were captured using a microscope (BZ9000). The cross-sectional area (CSA) was analyzed using Fiji software. The size distribution was evaluated. The average number of central nuclei in regenerated myofibers of the TA muscle was also evaluated for fusion efficiency during regeneration.

\section{Immunohistochemistry}

Cryosections (9- $\mu \mathrm{m}$ thickness) were made from injured and intact TA muscle. They were fixed with $4 \%$ paraformaldehyde for $15 \mathrm{~min}$ at $25^{\circ} \mathrm{C}$. After washing with phosphate-buffered saline (PBS), the sections were blocked with a blocking buffer containing 3\% BSA, 5\% goat serum, and $0.5 \%$ Triton-X for $30 \mathrm{~min}$. After several washes with $\mathrm{PBS}$, the sections were incubated with M.O.M. blocking reagent for $45 \mathrm{~min}$ at $25^{\circ} \mathrm{C}$ (Vector Laboratories Inc., Burlingame, CA, USA). After washing with PBS, they were incubated with primary antibody against Pax7 (1:10, clone Pax7; DSHB, University of Iowa, Iowa City, IA), myogenin (1:10, BD Pharmingen, San Jose, CA, USA), and polyclonal anti-laminin (1:200; Sigma Chemical Co., St. Louis, MO, USA) in staining solution (Can get signal hist A; Toyobo Co. Ltd., Osaka, Osaka, Japan) for $90 \mathrm{~min}$ at $25^{\circ} \mathrm{C}$. After washing with PBS, the slides were incubated with Alexa Fluor 488 (antirabbit)-conjugated and 568 (antimouse)-conjugated secondary antibody (1:1,000, Thermo Fisher Scientific K.K.) for $30 \mathrm{~min}$ at $25^{\circ} \mathrm{C}$ followed by incubation with DAPI solution $(0.01 \mathrm{mg} / \mathrm{mL}$ in $\mathrm{PBS})$ for $1 \mathrm{~min}$. After washing, the slides were mounted using a fluorescence medium (AquaPoly/Mount; Polysciences Inc., Warrington, PA, USA) and 
were visualized using a digital microscope (BZ-X9000) and were analyzed using Fiji software.

\section{Western blotting}

Samples were homogenized in an ice-cold buffer $(50 \mathrm{mM}$ Tris-Cl, $200 \mathrm{mM} \mathrm{NaCl}, 50 \mathrm{mM} \mathrm{NaF}, 0.3 \%$ NP-40, pH 8.0) with protease and phosphatase inhibitors (Nacalai Tesque Inc., Chukyo-ku, Kyoto, Japan). The protein concentration was measured using the bicinchoninic acid (BCA) method (BCA assay kit; Thermo Fisher Scientific K.K.). An equal amount of protein was separated using standard SDSPAGE and was transferred to a PVDF membrane. The membrane was blocked with a blocking reagent (Blocking One; Nacalai Tesque Inc.) and was incubated with primary antibodies. The primary antibodies used were phosphorylated Tyr ${ }^{641}$-STAT6 (1:1000, \#9362; Cell Signaling Technology Inc., MA, USA), STAT6 (1:2000, \#6778; Cell Signaling Technology Inc.), phosphorylated $\mathrm{Thr}^{180 /}$ Tyr182-p38 Mitogen-activated protein kinase (MAPK; 1: 1000, \#4511; Cell Signaling Technology Inc.), p38 MAPK (1:1000, \#9212; Cell Signaling Technology Inc.), and glyceraldehyde-3-phosphate dehydrogenase (GAPDH; 1: 2000, \#2118; Cell Signaling Technology Inc.). Luminescence signals by ECL reagent (Bio-rad Laboratories Inc., Hercules, CA, USA) were captured using an imaging system (LAS-4000; Fujifilm Corp., Minato-ku, Tokyo, Japan). Densitometry analysis was conducted using Fiji software.

\section{Quantitative reverse transcription-polymerase chain reaction (QRT-PCR)}

Details of the protocol have been described in an earlier report [24]. Total RNA from the cells was isolated using RNA extraction reagent (Sepasol-RNA I Super G; Nacalai Tesque Inc.) and RNA mini-columns (FATRK 001; Favorgen Biotech Corp., Ping-Tung, Taiwan) according to the manufacturers' protocols. The first-strand cDNA for PCR was generated using a commercially available kit (FSQ-301; Toyobo Co. Ltd.). Quantification of mRNA expression was performed using a real-time PCR system (Step One Plus; Life Technologies Japan Ltd., Minato-ku, Tokyo) with Syber green master mix reagent (QPS-101; Toyobo Co. Ltd.). For delta-delta Ct analysis, $\beta$-Actin or GAPDH mRNA was used as an internal reference. The primer sequences used for this study are presented in the Supplementary Table.

\section{Indirect cell number measurement}

Adenovirus-infected cells were grown for 24 and $48 \mathrm{~h}$ in GM. Cells at each time point were counted (cell counting kit-8 \#CK04; Dojindo Laboratories, Kamimashiki-gun, Kumamoto, Japan) according to the manufacturer's instructions. The indirect cell number was expressed with absolute absorbance at $450 \mathrm{~nm}$ (Multiskan MS; Life Technologies Inc.). The proliferation rate was calculated as the cell number at $48 \mathrm{~h}$ divided by the cell number at $24 \mathrm{~h} \mathrm{[26].}$

\section{Statistical analyses}

Data are presented as mean \pm standard deviation (SD). One-way analysis of variance (ANOVA) followed by the Tukey-Kramer multiple comparisons test was used to assess multiple group data. Unpaired $t$ tests were used for comparisons between the two groups. All analyses were performed using software (Prism v.8.0; GraphPad Software Inc., CA, USA). Significance was inferred for $p<.05$.

\section{Results}

STAT6 is deactivated during myotube formation, but IL-4 stimulates STAT6 activity

We first isolated myoblasts from WT mice and incubated the cells in DM for 24 and $48 \mathrm{~h}$ myotube formation to investigate phosphorylated-STAT6 (p-STAT6) (Fig. 1a). Results showed that p-STAT6 expression decreased at $24 \mathrm{~h}$ after adding DM, which was significant at $48 \mathrm{~h}$. We next examined the effects of IL-4 on STAT6 activation. After the cells were treated with IL-4 during DM incubation for $48 \mathrm{~h}$, we assessed the p-STAT6 expression in those cells. Results showed that p-STAT6 had been increased significantly by IL-4 treatment, indicating that STAT6 is a downstream target for IL-4 during myotube formation (Fig. 1b). These results indicate that STAT6 activity is decreased during normal myogenesis, where endogenous IL4 is expected to stimulate STAT6 activation [21, 24].

\section{STAT6 overexpression impairs myoblast fusion}

After finding that STAT6 can be activated by IL-4 during myotube formation, we aimed to clarify whether STAT6 can be involved in myogenesis. Because IL-4 is known to contribute to myoblast fusion, we first specifically examined the link between STAT6 and myoblast fusion. STAT6 was overexpressed in myoblasts by the adenoviral vector. At $24 \mathrm{~h}$ after infection, the myoblasts were induced to differentiate by the DM for $48 \mathrm{~h}$. Western blot results confirmed that STAT6 protein expression increased significantly (Fig. 2a and b), indicating that STAT6 was induced successfully. Phosphorylated-STAT6 levels also exhibited the same trend as those of total STAT6 protein (Fig. 2c). We performed immunohistochemical analysis to examine myotube formation (Fig. 2d). The fusion index (Fig. 2e) and diameter (Fig. 2f) of cells overexpressing STAT6 were significantly lower than those of control cells. STAT6 overexpression increased the percentage of myosin-positive cells possessing a single nucleus significantly but decreased the percentage of cells possessing three or more nuclei (Fig. 2g). These results indicate that overexpression of STAT6 impairs myoblast fusion. 

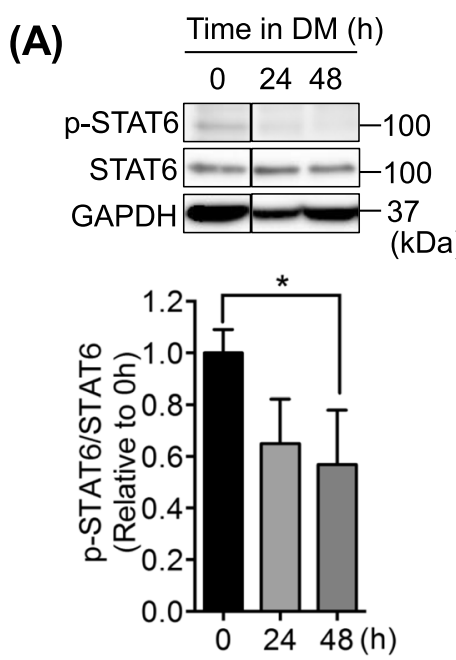

(B)
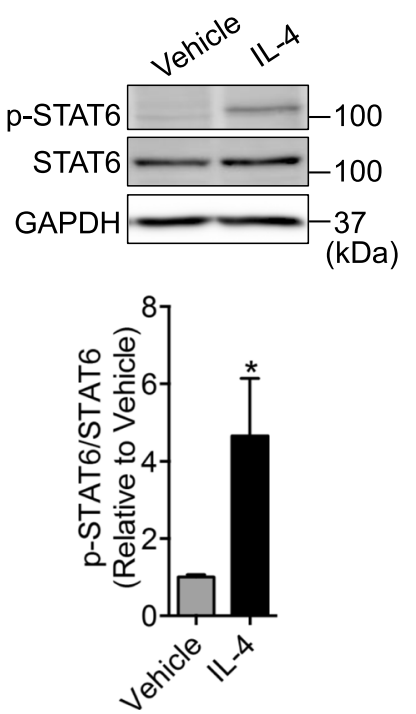

Fig. 1 STAT6 was deactivated during myogenesis and IL-4 increased STAT6 phosphorylation. a PSTAT6 expression during differentiation. Representative images show Western blot bands for phosphorylated STAT6 (p-STAT6), STAT6, and glyceraldehyde-3-phosphate dehydrogenase $(\mathrm{GAPDH}) n=3 .{ }^{*} p<.05$ vs. 0 h. b Phosphorylation of STAT6 after IL-4 treatment in cultured myotubes. Representative images of Western blot bands for $\mathrm{p}$-STAT6, STAT6, and GAPDH. $n=3 .{ }^{*} p<.05$ vs. $0 \mathrm{~h}$. Data are presented as mean \pm standard deviation (SD)

(A)

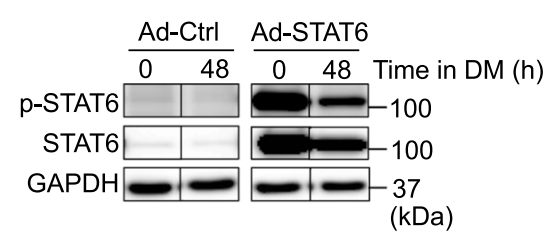

(D)
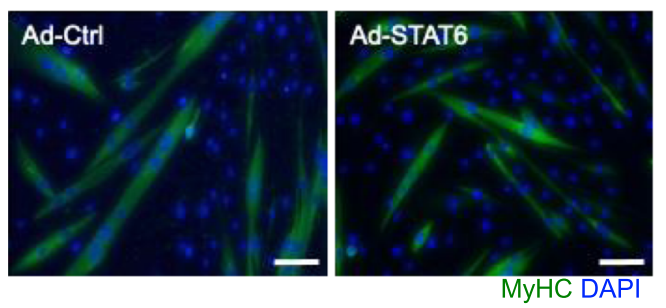

(B)

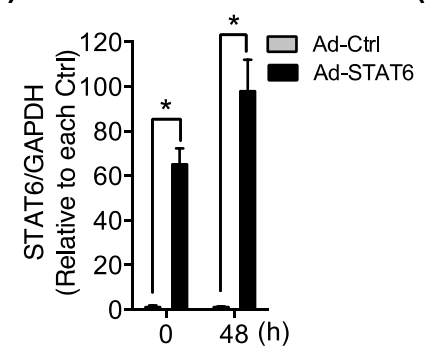

(E)

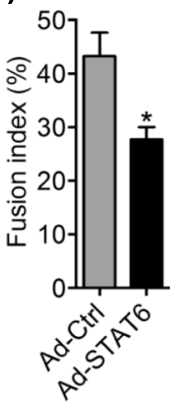

(C)

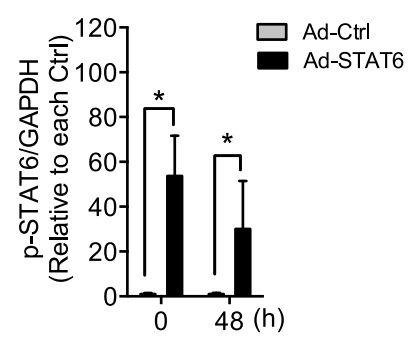

(G)

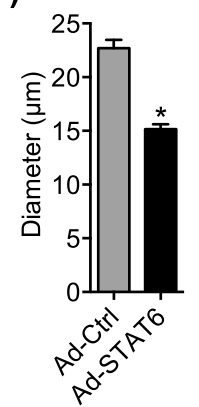

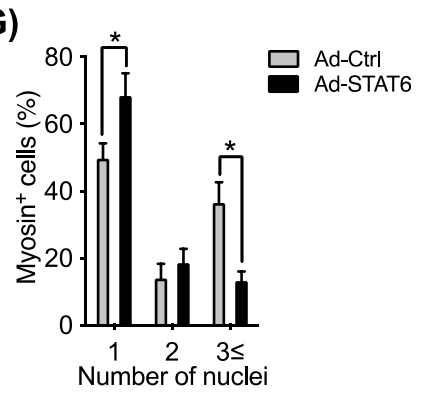

Fig. 2 STAT6 overexpression impaired myoblast fusion. a Representative images of Western blot bands for p-STAT6 and GAPDH. Myoblasts were infected with Ad-Ctrl or Ad-STAT6 adenovirus vector in growth medium (GM). After 24 h, the medium was replaced with a differentiation medium and maintained for $48 \mathrm{~h}$. The cells were then used for Western blot and immunocytochemical analysis. b Relative expression of total STAT6. $n=6 .{ }^{*} p<.05$ vs. Ad-Ctrl. c Relative expression of phosphorylated STAT6. $n=6 .{ }^{*} p<.05$ vs. Ad-Ctrl. d Representative immunostained myotubes positive for MyHC (green). Nuclei were stained with DAPI (blue). Scale bar $=50 \mu \mathrm{m}$. e Calculation of the fusion index in the Ad-Ctrl and Ad-STAT6 treatments. $\mathbf{f}$ Diameters of myotubes in the Ad-Ctrl and Ad-STAT6 treatments. $\mathbf{g}$ Percentages of myosin-positive cells with one, two, and three or more nuclei. $n=6$ in each group. ${ }^{*} p<.05$ vs. Ad-Ctrl. Data are presented as mean \pm SD 


\section{STAT6 knockdown improves myoblast fusion}

Given that STAT6 deactivation is necessary during myotube formation, we hypothesized that the inhibition of STAT6 promotes myoblast fusion. To test this hypothesis, the myoblasts were transfected using a shSTAT6 vector to knockdown STAT6 and was maintained for 48 $\mathrm{h}$ in GM. Control myoblasts were transfected with an empty vector (i.e., Ctrl). After $48 \mathrm{~h}$, the medium was switched to DM. Then incubation continued for $48 \mathrm{~h}$. Based on Western blot (Fig. 3a), we confirmed that the expression of STAT6 (Fig. 3b) and of p-STAT6 (Fig. 3c) decreased significantly after shRNA transfection at 0 and $48 \mathrm{~h}$ of incubation in DM. We then conducted an immunohistochemical analysis to find the levels of myotube formation (Fig. 3d). The fusion index was modest but significantly higher in STAT6-knocked-down cells than in Ctrl cells (Fig. 3e). The diameter in STAT6knocked-down cells was significantly higher than that in Ctrl cells (Fig. 3f). The percentage of myosin-positive cells possessing a single nucleus decreased, although the percentage of cells possessing three or more nuclei increased significantly in STAT6-knocked-down cells (Fig. $3 g)$. Collectively, these results suggest an inhibitory role of STAT6 in myoblast fusion.
STAT6 implicates gene expression related to myoblast fusion

Several molecules are known to be associated with myoblast fusion. We next tested whether those fusion-related molecules might be affected by the experimental modulation of STAT6. The respective mRNA expressions of myomaker [28], myomerger [29], Adam12 [30], $\beta 1 D$-integrin [31], $\beta 1$-integrin [32], M-cadherin [33], N-cadherin [34], caveolin-3 [35], and myoferin [36] were compared with myotubes differentiated for $48 \mathrm{~h}$ between Ad-Ctrlinfected and Ad-STAT6-infected cells. As portrayed in Fig. 4a, many molecules tended to be lower. Myomaker and myomerger were decreased significantly in Ad-STAT6 cells. By contrast, myomaker, myomerger, $\beta 1 D$-integrin, and caveolin-3 mRNAs were increased significantly in STAT6-knocked-down cells compared to those in Ctrl cells (Fig. 4b). These results were comparable to results of morphological analysis shown in Figs. 2 and 3.

\section{STAT6 affected the differentiation of myoblasts}

Because STAT6 manipulation was performed in myoblasts, we next investigated whether STAT6 is also involved in myoblast differentiation and proliferation as
(A)

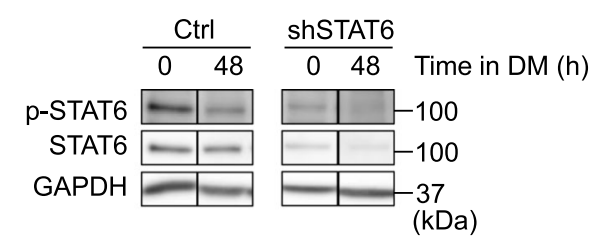

(D)

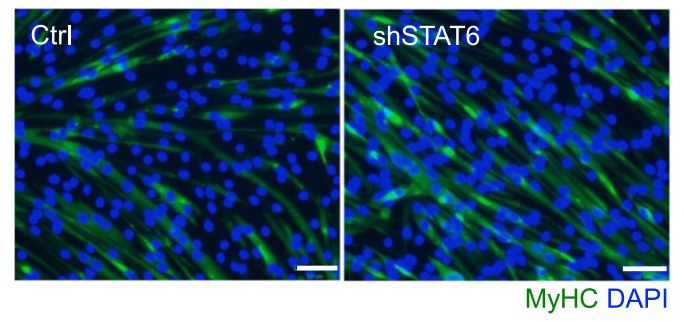

(B)

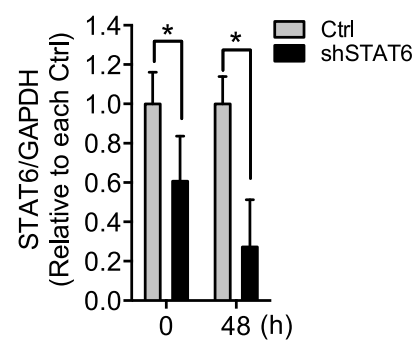

(E)

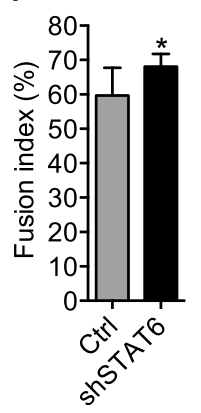

(F)
(C)

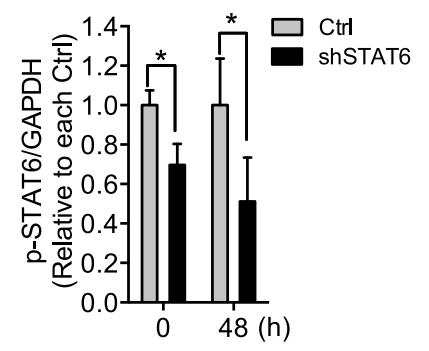

(G)

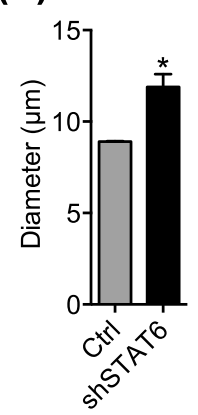

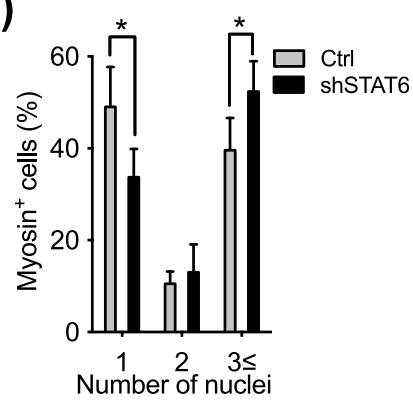

Fig. 3. STAT6 knockdown improved myoblast fusion. Myoblasts were transfected with empty (Ctrl) or shSTAT6 vectors and grown in GM. After 48 $\mathrm{h}$, the cells were harvested to ascertain p-STAT6, STAT6, and GAPDH levels via Western blotting. a representative images of Western blot bands. Relative protein expression levels of $\mathbf{b}$ STAT6 and $\mathbf{c} p$-STAT6. $n=6$ per group. ${ }^{*} p<.05$ vs. Ctrl. $\mathbf{d}$ representative immunostained myotubes positive for MyHC (green). Nuclei were stained with DAPI (blue). Scale bar $=50 \mu \mathrm{m}$. e Calculation of fusion index in the Ctrl and shSTAT6 treatment. $n=4$. $\mathbf{f}$ Diameters of myotubes in the Ctrl and shSTAT6 treatments. $\mathbf{g}$ Percentages of myosin-positive cells. $n=4$. ${ }^{*} p<.05$ vs. Ctrl. Data are presented as mean \pm SD 


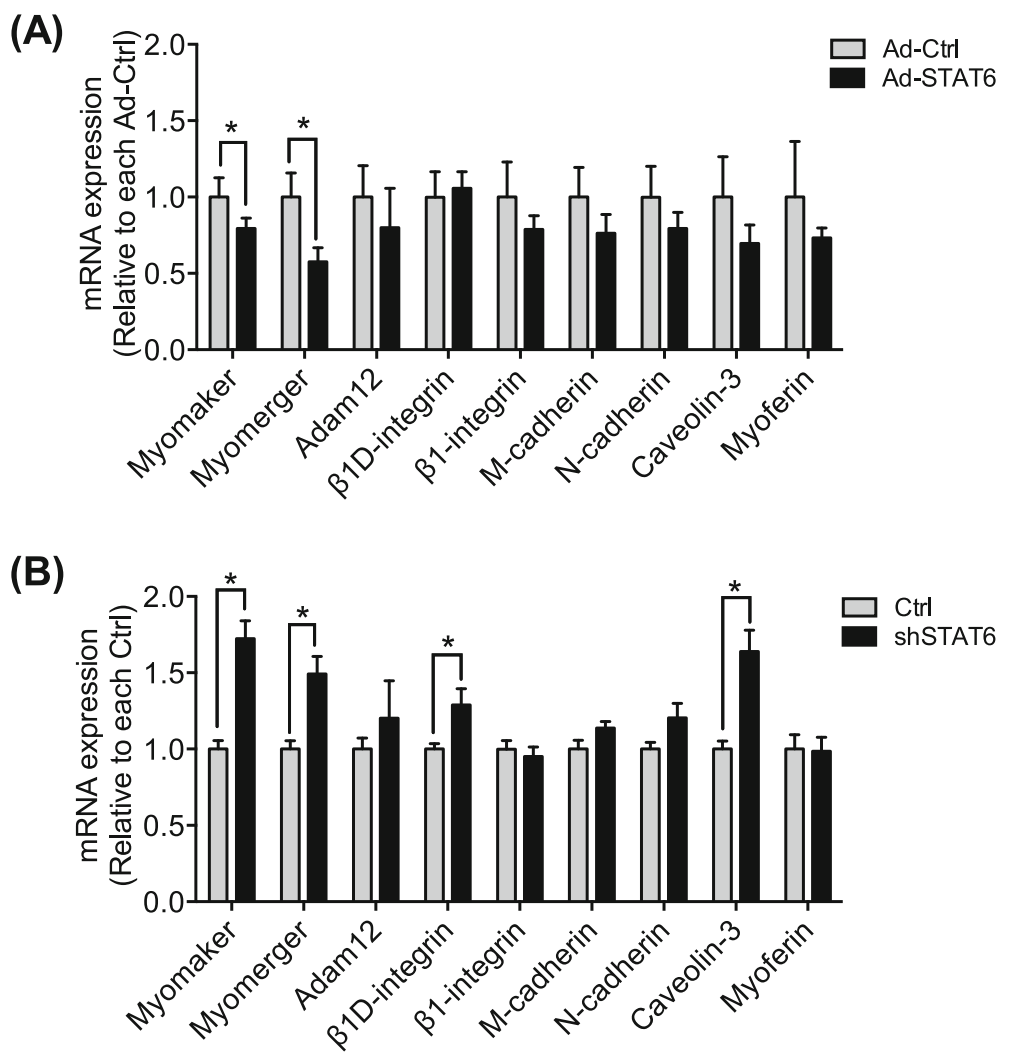

Fig. 4 Expression of mRNA related to myoblast fusion in STAT6-overexpressed and STAT6-knockdown cells. a mRNA expression in the Ad-Ctrl and Ad-STAT6 treatments. $n=4$ per group. ${ }^{*} p<.05$ vs. Ctrl. $\mathbf{b}$ mRNA expression in the Ctrl and shSTAT6 treatments. $n=6$ per group. ${ }^{*} p<.05$ vs. Ctrl. Data are presented as mean \pm SD
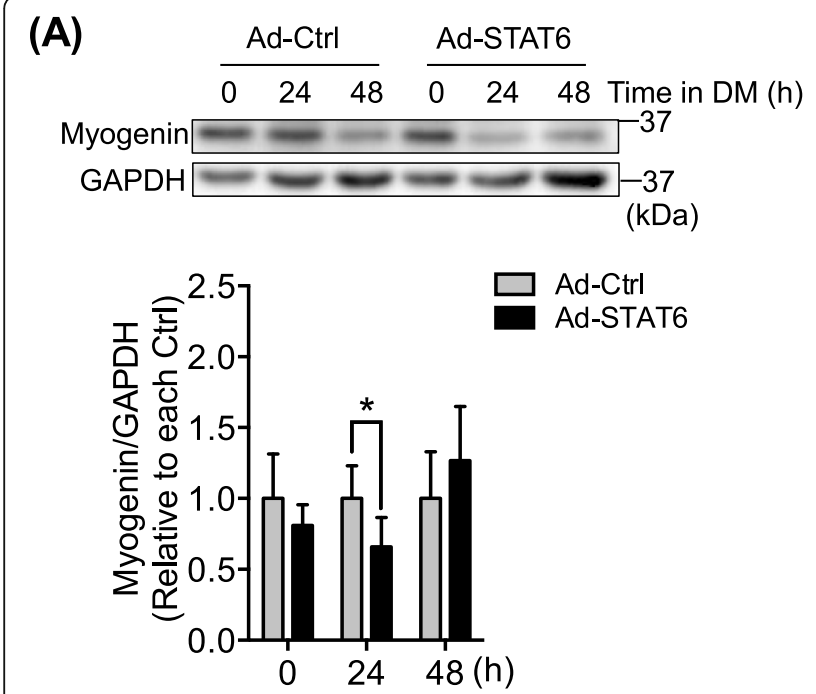

\section{Ad-Ctrl}

Ad-STAT6
(B)
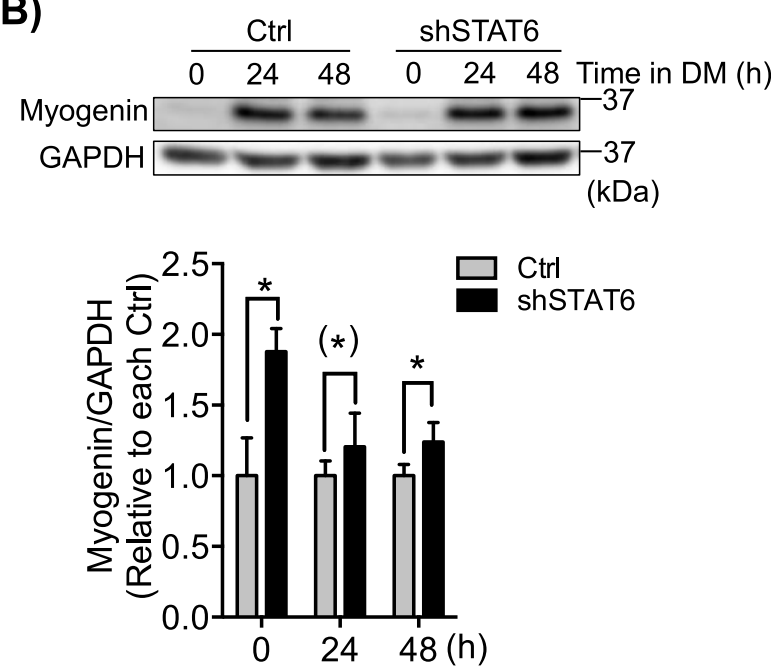

Fig. 5 Intervention for STAT6 influences differentiation in culture. a Western blot for myogenin and GAPDH in the Ad-Ctrl and Ad-STAT6 treatments. $n=6 .{ }^{*} p<.05$ vs. Ad-Ctrl. b Western blot for myogenin and GAPDH in the Ctrl and shSTAT6 treatments. $n=6 .{ }^{*} p<.05$ vs. Ctrl. $\left({ }^{*}\right) p$ $=.08$ vs. Ctrl. Data are presented as mean \pm SD 
part of prefusion events. First, myogenin expression was analyzed during DM incubation for 0,24 , and $48 \mathrm{~h}$ in Ad-Ctrl and Ad-STAT6 infected cells. The adenoviral infection seemed to induce myogenin expression at $0 \mathrm{~h}$, but the expression was found to have no significant difference between Ad-Ctrl and Ad-STAT6 (Fig. 5a). The myogenin expression was decreased significantly in AdSTAT6 cells after $24 \mathrm{~h}$ of DM incubation (Fig. 5a). No difference was found at $48 \mathrm{~h}$ (Fig. 5a). On the other hand, at 0 and after $48 \mathrm{~h}$ of DM incubation, myogenin expression was increased significantly in STAT6knocked-down cells compared to in Ctrl cells (Fig. 5b). A similar result was obtained at $24 \mathrm{~h}(p=.08)$. Although the time course change varied between gain-of-function and loss-of-function conditions, these results suggest that STAT6 was implicated in myogenin expression as an inhibitory factor during DM incubation. We next analyzed the differentiation index in an identical sample used for fusion index analysis in Figs. 2 and 3. Results show that the differentiation index was decreased significantly in AdSTAT6 myotubes (Fig. S1A) but that it was increased significantly in STAT6-knocked-down myotubes (Fig. S1B). Considered collectively, these results suggest that STAT6 plays inhibitory roles in the differentiation program.

We also tested the effects of overexpression of STAT6 on myoblast proliferation. Results show no difference in the indirect cell number or proliferation rate between Ad-Ctrl and Ad-STAT6 myoblasts (Fig. S2A-S2C). Therefore, STAT6 was not suggestive of implicating myoblast proliferation.

\section{STAT6-KO mice exhibit improved regeneration after injury}

We examined whether the absence of STAT6 affects adult regenerative myogenesis in vivo, or not. No difference was found in the body mass of WT or STAT6-KO mice studied (Fig. 6a). Cardiotoxin in saline was injected into the left TA muscles of WT and STAT6-KO mice to induce muscle injury, followed by regeneration. At 5 days post-injection, no difference was found in fiber CSA distribution in the right intact TA (Fig. 6b and c). However, in the regenerated TA, the percentage of myofibers between 300 and $600 \mu \mathrm{m}$ was significantly lower, whereas the percentage of myofibers of more than 900 $\mu \mathrm{m}$ was significantly higher in STAT6-KO than in WT mice (Fig. $6 \mathrm{~b}$ and d). The average number of central nuclei in regenerating myofibers of the TA muscle was found to be significantly higher in STAT6-KO than in WT mice (Fig. 6e). Next, mRNA expression of pax7, myogenin, myomaker, embryonic $\mathrm{MyHC}(\mathrm{eMyHC})$, and IL-4 was examined in the regenerated TA muscle. No change in pax7 or myomaker mRNA, myogenin or

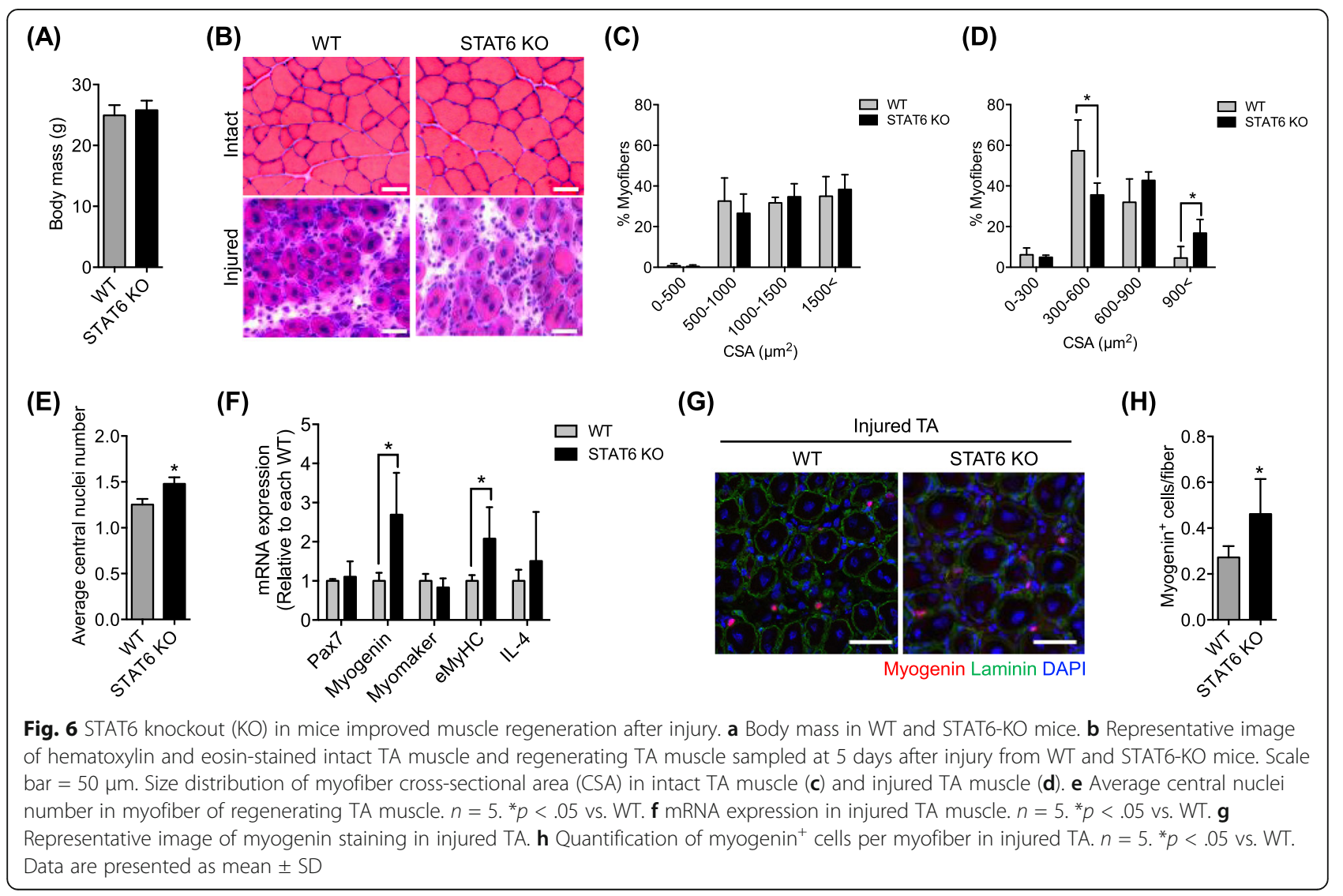


eMyHC mRNA was significantly higher in STAT6-KO than in WT mice (Fig. 6f). No difference was found in IL-4 mRNA between WT and STAT6-KO mice (Fig. 6f). We also made a cryosection of TA to examine myogenin ${ }^{+}$cell numbers using immunohistochemistry. Myogenin ${ }^{+}$cell numbers were significantly higher in STAT6-KO mice than in WT mice (Fig. $6 \mathrm{~g}$ and $\mathrm{h}$ ). Altogether, these results indicate that muscle regeneration after injury was facilitated in STAT6KO mice by enhancing myogenic differentiation and fusion. The comparable IL- 4 mRNA levels imply that the effects of IL-4 were comparable between WT and STAT6-KO mice irrespective of their regeneration discrepancy.

In a separate analysis, we examined $\operatorname{pax} 7^{+}$satellite cell numbers immunohistochemically (Fig. S3A). We found no difference between the numbers obtained for WT and STAT6-KO mice (Fig. S3B). In light of the results of cell number analysis shown in Fig. S2, we infer that STAT6 does not influence myogenic cell proliferation.

\section{Inhibitory action of STAT6 is independent of IL-4 signaling}

Using the STAT6-KO mice model, we then sought to elucidate whether STAT6 can mediate IL-4-induced stimulation of myotube formation. The myoblasts isolated from WT and STAT6-KO mice were differentiated by DM incubation for $48 \mathrm{~h}$ with or without IL-4. Then, the myotubes were fixed and stained with $\mathrm{MyHC}$ and DAPI to examine the fusion index (Fig. 7a). Results indicate that IL-4 significantly increased the fusion index in WT cells (Fig. 7b). The fusion index in STAT6-KO cells was significantly higher than that in WT cells irrespective of IL-4 treatment (Fig. 7b). It is particularly interesting that the fusion index in STAT6-KO cells with IL-4 treatment was significantly higher than that in WT cells with IL-4 treatment. These results suggest that IL-4 and STAT6 independently implicate myotube formation. This finding implies that STAT6 did not mediate a positive role of IL-4 in myotube formation.

To ascertain the reasons for the separate influence of IL-4 and STAT6, we sought candidate molecules for regulating myogenesis via STAT6. During this process, we found a discrepancy in p38 MAPK, which is an essential kinase for myogenesis [37], in those cells by Western blot (Fig. 7c). As expected, IL-4 increased p-STAT6 in WT cells significantly (Fig. 7d). Also, STAT6 was not detected in STAT6-KO cells (Fig. 7d). In this situation, p-

\section{(A)}
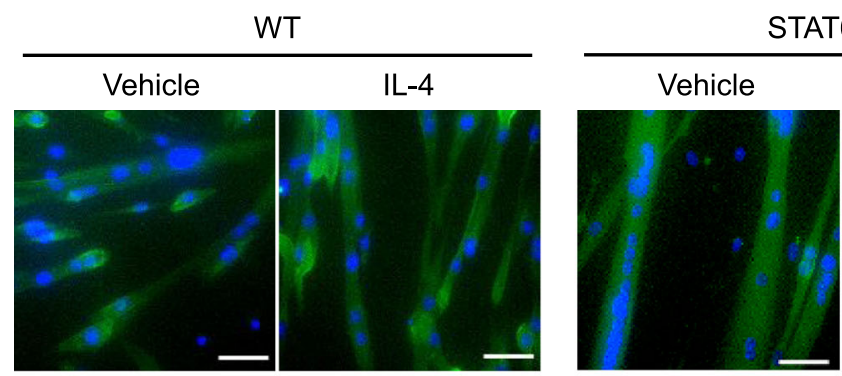

STAT6 KO

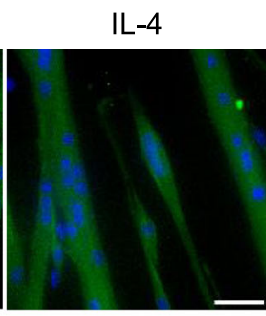

MyHC DAPI

(C)

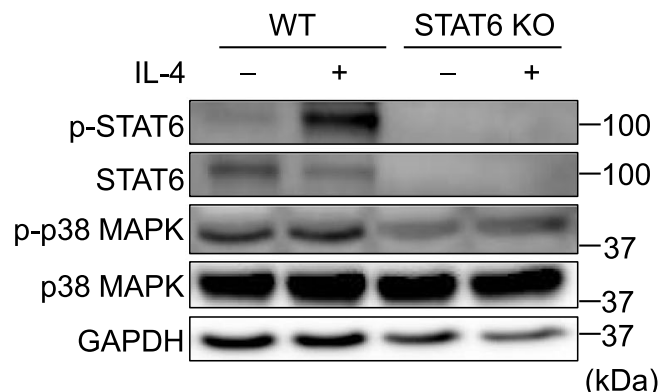

(D)

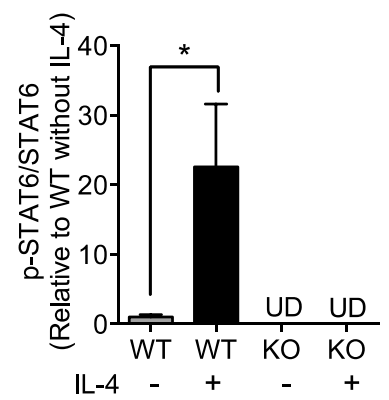

(B)

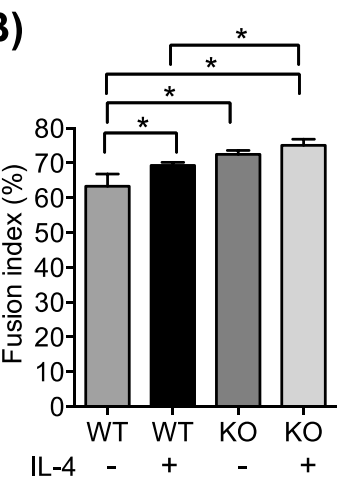

(E)



Fig. $7 \mathrm{IL}-4$ and STAT6 independently regulated myogenesis. Myoblasts were isolated from WT and STAT6-KO mice and maintained in DM with or without IL-4 treatment. a Representative immunostained myotubes positive for MyHC (green). Nuclei were stained with DAPI (blue). Scale bar = $50 \mu \mathrm{m}$. b Quantification of fusion index. $n=5$. * $p<.05$. c Representative protein bands for p-STAT6, STAT6, p-p38 MAPK, p38 MAPK, and GAPDH. d p-STAT6 protein expression. $n=6 .{ }^{*} p<.05$ by unpaired $t$ test. UD denotes undetected. e p-p38 MAPK protein expression. ${ }^{*} p<.05$. Data are presented as mean \pm SD 
p38MAPK in STAT6-KO cells was significantly decreased compared to that in WT cells, irrespective of IL-4 treatment (Fig. 7e). These results implicate p38 MAPK in STAT6 during myotube formation. Considering the results obtained for the fusion index (Fig. 7b), that the repressive role of STAT6 in myotube formation is mediated by p38 MAPK activity, independent of IL-4 action.

\section{Myoblast-myotube fusion is attenuated in STAT6- overexpressed myoblasts}

We further examined the possibility of an unlikely mediation of STAT6 in IL-4-induced myotube formation. It has been established that IL-4 signaling is important for myoblasts to fuse with myotubes [21]. Therefore, it would be expected that myoblast-myotube fusion can be enhanced by STAT6 activation if the IL-4-STAT6 axis served as a stimulatory pathway for it. To this end, we followed an earlier reported protocol but used
STAT6 overexpression instead of IL-4R $\alpha \mathrm{KO}$ in myoblasts [21]. Isolated $\mathrm{mTFP}^{+}$myoblasts expressing STAT6 normally were pre-incubated in DM for $48 \mathrm{~h}$ to form $\mathrm{mTFP}^{+}$myotubes. Concomitantly, the WT myoblasts were infected with either Ad-STAT6 or Ad-Ctrl expressing RFP. These $\mathrm{RFP}^{+}$myoblasts were then added to $\mathrm{mTFP}^{+}$myotubes and were maintained for the next $48 \mathrm{~h}$ to induce fusion of $\mathrm{RFP}^{+}$myoblasts to $\mathrm{mTFP} 1^{+}$ myotubes (Fig. 8a). The numbers of $\mathrm{mTFP}^{+}$mononuclear (i.e., unfused) cells were comparable between Ad-STAT6 and Ad-Ctrl cultures (Fig. 8b), suggesting that their myotube conditions were comparable. The chimeric myotubes, expected mostly to signify the fusion of adenovirus-infected myoblasts with myotubes, were significantly fewer in the Ad-STAT6 treatment (Fig. 8c). The number of unfused $\mathrm{RFP}^{+}$mononuclear cells was significantly higher in the Ad-STAT6 than in the Ad-Ctrl culture (Fig. 8d). Therefore, although this experimental

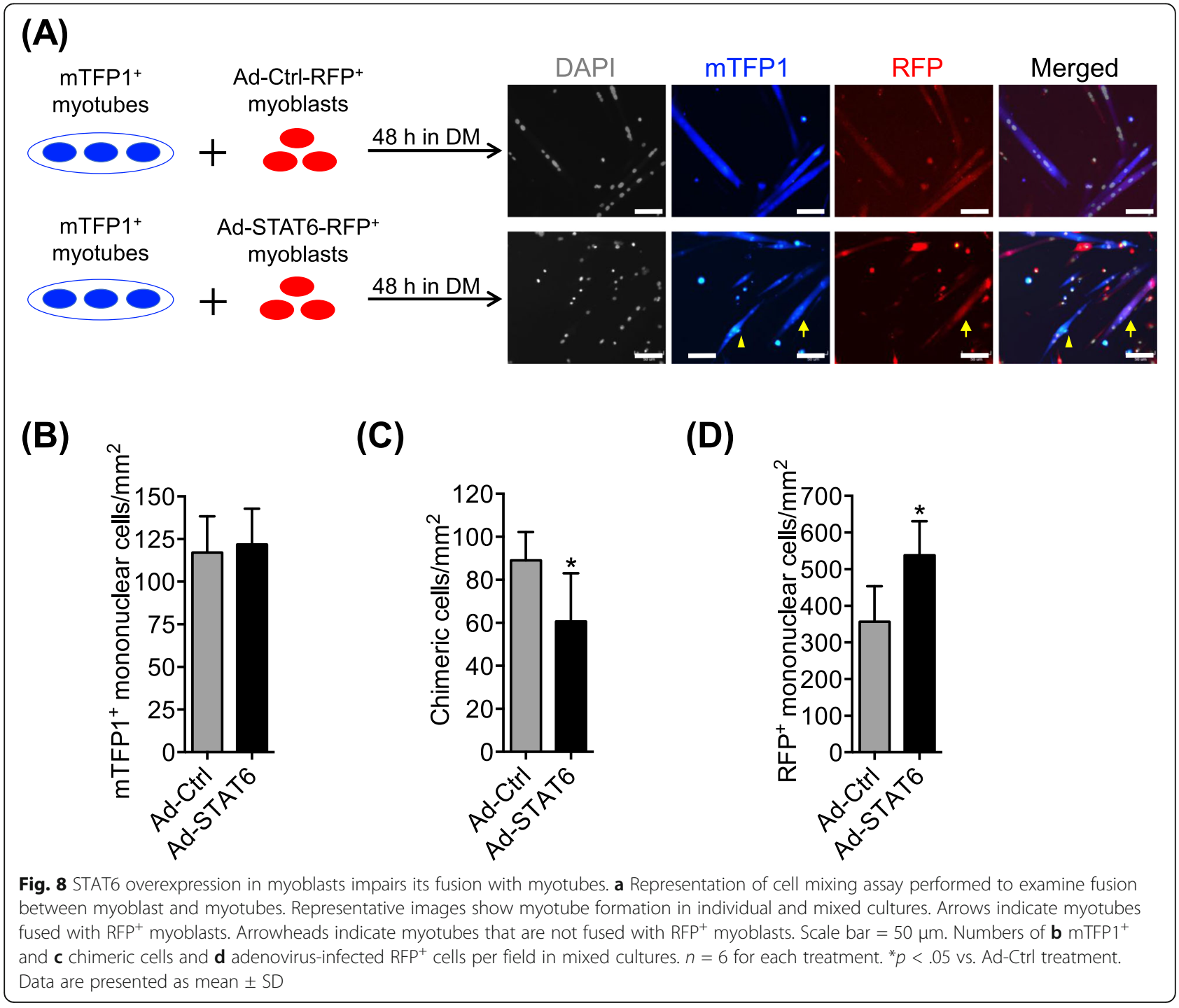


procedure can not entirely eliminate the possibility of myoblast-myoblast fusion, results seem to support the notion that STAT6 does not mediate IL-4-linked promotion of myoblast fusion.

\section{Discussion}

Mechanisms underlying myoblast differentiation and fusion remain unclear. Our first-time gain-of-function and loss-of-function experiments demonstrated that overexpression of STAT6 inhibits, whereas excessive inhibition of STAT6 improves, myoblast differentiation and fusion in vitro. In vivo experiments using STAT6-KO mice demonstrated that the muscle regeneration process was improved in the absence of STAT6 expression. Results also demonstrate that inhibition of myogenesis by STAT6 might not be associated with IL-4. These results suggest that STAT6 is a protein that negatively regulates adult myogenesis. Figure 9 depicts a putative scheme of these study results.

Results of earlier studies have shown that IL-4 facilitates fusion in cultured myocytes $[19,21]$ or that IL-4 promotes myogenic differentiation in colon carcinomabearing mice [38]. Earlier studies in nonmuscle cell types have demonstrated that STAT6 is activated primarily by IL-4 $[12,13]$. Therefore, we speculated that STAT6 is involved in the muscle regeneration process as a positive regulator. However, although we found that IL-4 can activate STAT6 during myogenesis, results showed that STAT6 had an inhibitory rather than a stimulatory effect on muscle formation. Indeed, STAT6 activity was decreased during DM incubation. Results suggest that the deletion of STAT6 and IL-4 treatment independently improved the fusion index in culture. Moreover, STAT6-overexpressed myoblasts showed a lower capacity to fuse with myotube under culture conditions. In addition, whereas IL- $4 \mathrm{KO}$ and IL-4R $\alpha \mathrm{KO}$ mice did not exhibit improved CSA at 8 days of post injury [21], we observed a greater CSA at 5 days post injury in STAT6$\mathrm{KO}$ mice than in WT mice. Altogether, these findings suggest that the IL-4-STAT6 signaling axis is not responsible for myotube formation. Other mechanisms are expected to control STAT6 activity to attenuate myogenesis. Based on results of earlier studies, multiple molecules are potentially involved in myogenesis, which can regulate STAT6 in cellular events. For instance, the mammalian target of rapamycin (mTOR) inhibits STAT6 activity in T cells [39]. Also, mTOR-signaling activation is important for myogenesis [40, 41]. Consequently, mTOR activation might inhibit STAT6 signaling during myotube formation. Alternatively, interferon$\beta$ can activate STAT6 in hepatoma cells [42]. Because interferon- $\beta$ impairs myotube formation [43], interferon-

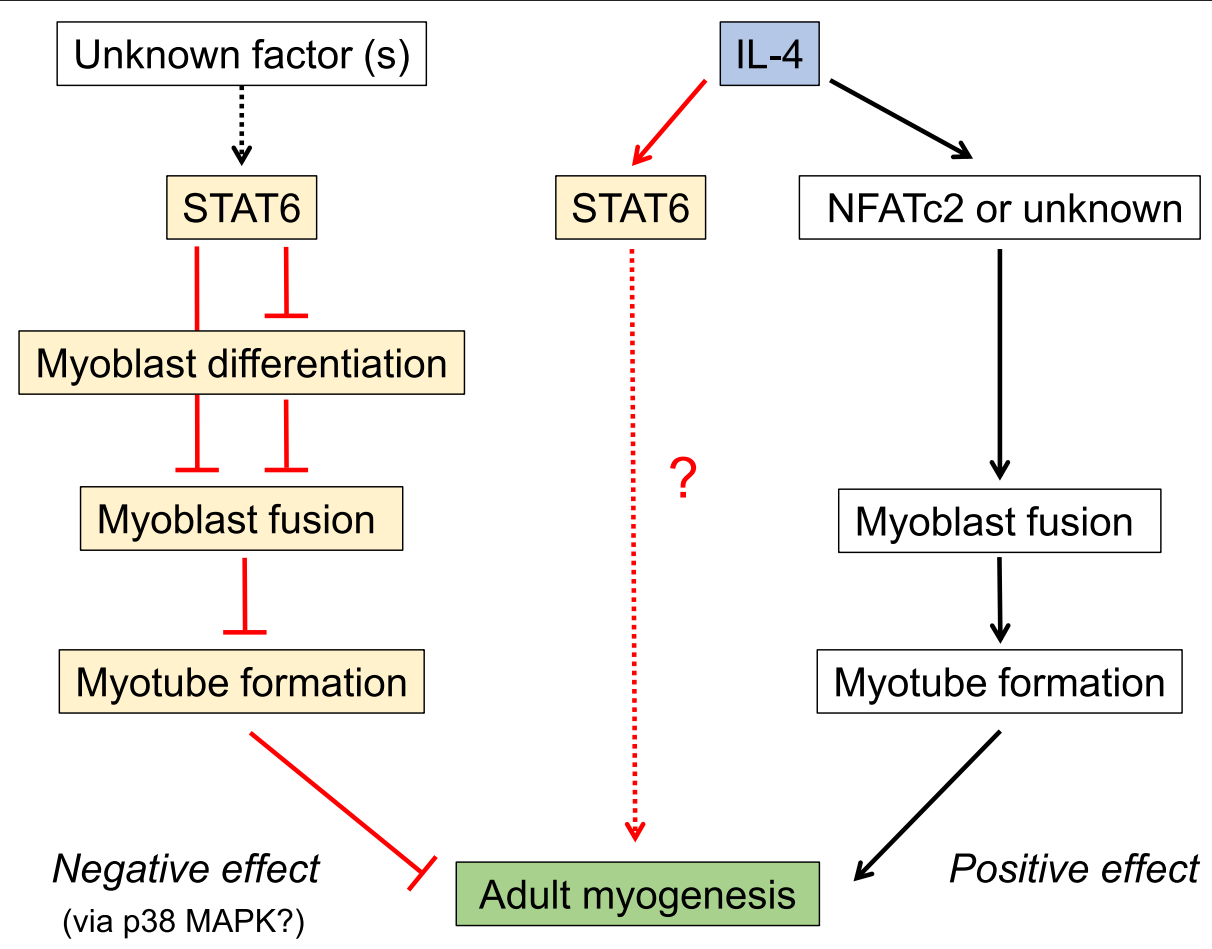

Fig. 9 Putative roles of STAT6 in adult myogenesis. IL-4 can activate STAT6 in myotubes. However, STAT6 inhibits myotube formation. IL-4 improves myoblast fusion independently of STAT6 activity. The IL-4-STAT6 signaling axis therefore does not account for IL-4 related myotube formation processes. The red line is based on results from this study. The black line shows data referred from earlier studies. The dashed line represents unvalidated data 
$\beta$ signaling might negatively regulate myogenesis via STAT6. Some molecules such as interferon- $\alpha$ [42, 44], IL-13 [45], and leptin [46] interact with STAT6 in nonmuscle cell types. Eventually, further study will be necessary to identify molecules that control STAT6 activity in myogenesis as an inhibitory factor.

We observed an IL-4-independent decrease in p38 MAPK activity in fusion-facilitated STAT6-KO myotubes. This decrease suggests a possible relation between STAT6 and p38 MAPK during myogenesis. Actually, the role of p38 MAPK in myogenesis is complicated: p38 MAPK is necessary to execute timely myogenic differentiation by activating myocyte enhancer factor 2 (MEF2) [47] or MyoD and its co-molecule E47 [37]. Also, p38 MAPK mediates inhibition of myogenic cell cycling as a molecular switch to regulate myogenic commitment [48]. These results suggest that p38 MAPK contributes to advancement of myotube formation. By contrast, an inhibitory role of p38 MAPK in myogenesis has also been suggested. Suelves et al. has demonstrated that inhibition of p38 MAPK activity increased desmin and $\alpha-$ actin expression in $\mathrm{C} 2 \mathrm{C} 12$ myoblast differentiated for 5 days [49]. Contrary to an earlier report by Llouis et al. [37], results of at least one study show that p38 MAPK might mediate the inhibition of E47 activity by mitogen and extracellular kinase kinase 1 during differentiation in $\mathrm{C} 2 \mathrm{C} 12$ cells [50]. Moreover, Weston et al. has shown that p38 MAPK inhibition activated myogenin promoter and increased myogenin and MEF2C gene expression in C2C12 cells [51]. They also demonstrated that p38 MAPK inhibition promoted myogenesis in the distal limb or proximal mesenchyme myoblasts [51]. Accordingly, findings obtained from the current study agree with those of the earlier study [52] because STAT6 inhibition or deletion stimulated differentiation and fusion with attenuated p38 MAPK activity. A specific link between STAT6 and p38 MAPK during myogenesis must be clarified.

Although our STAT6-KO animals exhibited interesting results, attention must be devoted to their interpretation. First, our mice lacked STAT6 globally and congenitally. Therefore, we cannot rule out the possibility of physiological compensation for the loss of STAT6. Moreover, because STAT6 is necessary for immune cell regulation $[12,13]$, some alteration in the systemic inflammatory situation can be expected. However, results from acute shRNA-based STAT6 knockdown in isolated and STAT6$\mathrm{KO}$ myoblasts experiments partially counter those concerns. Second, no significant difference was found in cross-sectional area between WT and STAT6-KO intact muscles, implying no developmental problem in the mice. Considering that STAT6 influences myogenic differentiation, it is expected that developmental defects would occur in STAT6-KO mice because considerable myogenesis occurs in the embryo. Although we currently have no explanation related to this point, it is noteworthy that molecular machinery might be largely shared but not be completely identical between de novo developmental and adult myogenesis [53-55].

The physiological significance of STAT6 inhibitory function during myogenesis remains unknown. One supposition is that STAT6 adjusts the proper timing of myoblasts for myogenic commitment. Myogenesis is tightly regulated by the sequential activation or deactivation of signaling cascades $[5,52]$. The dysregulation of the cascades impairs myofiber formation. Our results demonstrate that STAT6 activity was evident for predifferentiation. Then, activity levels decreased along with the induction of differentiation. We observed that STAT6 can affect myogenin expression. Consequently, STAT6 might prevent the progression of myoblast differentiation and fusion until the myoblasts enter a fusion-competent state. At this time, fusion is allowed to proceed by decreasing STAT6 activity. Considering this inference as true, our findings related to myogenesis promotion by STAT6 inhibition imply a harmful influence of intact myofiber formation under practical situations. Consequently, the influence of STAT6 inhibition over the entire adult myogenesis period, during which a mature myofiber is established, must be elucidated.

\section{Conclusion}

Results indicate that STAT6 plays an inhibitory role in myoblast differentiation and fusion in adults. Moreover, the results suggest that the facilitation of myotube formation by IL-4 is independent of STAT6. Consequently, STAT6 might be a clinical target to achieve efficient muscle formation.

\begin{abstract}
Abbreviations
ANOVA: Analysis of variance; DAPI: 4', 6-Diamidino-2-phenylinodole; DM: Differentiation medium; ECL: Enhanced chemiluminescence; GAPD H: Glyceraldehyde-3-phosphate dehydrogenase; GM: Growth medium; IL: Interleukin; MAPK: Mitogen-activated protein kinase; KO: Knockout; mTFP1: Monomeric teal fluorescent protein 1; PVDF: Poly-vinylidene difluoride; RFP: Red fluorescent protein; SDS-PAGE: Sodium dodecyl sulfate polyacrylamide gel electrophoresis; shRNA: Short hairpin RNA; siRNA: Small interfering RNA; STAT: Signal transducer and activator of transcription
\end{abstract}

\section{Supplementary Information}

The online version contains supplementary material available at https://doi. org/10.1186/s13395-021-00271-8

\footnotetext{
Additional file 1: Figure S1. Differentiation index in STAT6-

overexpressed and STAT6-inhibited cells. (A) Differentiation index in the Ad-Ctrl and Ad-STAT6 treatments. $n=5 .{ }^{*} p<.05$ vs. Ad-Ctrl. Images in Fig. 2 were used for analysis. (B) Differentiation index in the Ctrl and shSTAT6 treatments. $n=5$. ${ }^{*} p<.05$ vs. Ctrl. Images in Fig. 3 were used for analysis. Data are presented as mean \pm SD. Figure S2. Proliferation of STAT6-overexpressed myoblasts. (A) Representative bright-field images of myoblasts in Ad-Ctrl and Ad-STAT6 cells. Scale bar $=50 \mu \mathrm{m}$. (B) Absorbance at $450 \mathrm{~nm}$ in Ad-Ctrl and Ad-STAT6 cells using a CCK cell counting
} 
kit. (C) Proliferation rate in Ad-Ctrl and Ad-STAT6 cells. $n=6$. Data are presented as mean \pm SD. Figure S3. Pax7-positive cells in regenerating TA muscle of WT and STAT6-KO mice. (A) Representative images in CTXinjured TA muscle. Scale bar $=50 \mu \mathrm{m}$. (B) Quantification of pax $7^{+}$cells per myofiber. $n=5$. Data are presented as mean \pm SD. Supplementary Table S1. Primer sequences for QRT-PCR.

\section{Acknowledgements}

We gratefully acknowledge C. Kakehashi (St. Marianna University School of Medicine) for support with the maintenance of genetically modified mouse lines and Dr. T. Yoshihara (Juntendo University) for his constructive comments to the manuscript draft.

\section{Authors' contributions}

M.K. and Y.O. designed and performed experiments and analyzed the data. The manuscript was written by M.K., Y.O., S.S., K.K., and T.F. The author(s) read and approved the final manuscript.

\section{Funding}

We acknowledge the funding support from the Grants-in-Aid for Scientific Research (C) to M.K. (15K01633 18K10904, and 21K11428) and to Y.O. (18K10835).

\section{Availability of data and materials}

All data generated and analyzed during the study are available from the corresponding author upon reasonable request.

\section{Declarations}

\section{Ethics approval and consent to participate}

All animal experimental procedures were approved by the Committee for Animal Experimentation (No.1510012), St. Marianna University School of Medicine and the St. Marianna University Gene Recombination Experiment Safety Committee (No. 14008, TG181120-4)

\section{Consent for publication}

All authors read and approved the final manuscript and consented to its submission to the Skeletal Muscle.

\section{Competing interests}

The authors declare that they have no competing interests.

\section{Author details}

'Department of Physiology, St. Marianna University School of Medicine, Kawasaki, Kanagawa 216-8511, Japan. ${ }^{2}$ School of Kinesiology, The University of Louisiana at Lafayette, Lafayette, LA, USA. ${ }^{3}$ New Iberia Research Center, The University of Louisiana at Lafayette, New Iberia, LA, USA.

Received: 17 November 2020 Accepted: 18 May 2021

Published online: 29 May 2021

\section{References}

1. Yoon MS. mTOR as a key regulator in maintaining skeletal muscle mass. Front Physiol. 2017:8:788. https://doi.org/10.3389/fphys.2017.00788.

2. Levine S, Nguyen T, Taylor N, Friscia ME, Budak MT, Rothenberg P, et al. Rapid disuse atrophy of diaphragm fibers in mechanically ventilated humans. N Engl J Med. 2008;358(13):1327-35. https://doi.org/10.1056/ NEJMoa070447.

3. Chang NC, Rudnicki MA. Satellite cells: the architects of skeletal muscle. Curr Top Dev Biol. 2014;107:161-81. https://doi.org/10.1016/B978-0-12-416022-4. 00006-8.

4. Le Grand F, Rudnicki M. Satellite and stem cells in muscle growth and repair. Development. 2007;134(22):3953-7. https://doi.org/10.1242/dev. 005934

5. Yin $H$, Price $F$, Rudnicki MA. Satellite cells and the muscle stem cell niche. Physiol Rev. 2013;93(1):23-67. https://doi.org/10.1152/physrev.00043.2011.

6. Cheung TH, Rando TA. Molecular regulation of stem cell quiescence. Nat Rev Mol Cell Biol. 2013;14(6):329-40. https://doi.org/10.1038/nrm3591.
7. Blau HM, Cosgrove BD, Ho AT. The central role of muscle stem cells in regenerative failure with aging. Nat Med. 2015;21(8):854-62. https://doi. org/10.1038/nm.3918.

8. Hindi SM, Tajrishi MM, Kumar A. Signaling mechanisms in mammalian myoblast fusion. Sci Signal. 2013;6(272):re2.

9. Kim JH, Jin P, Duan R, Chen EH. Mechanisms of myoblast fusion during muscle development. Curr Opin Genet Dev. 2015;32:162-70. https://doi. org/10.1016/j.gde.2015.03.006.

10. Sampath SC, Sampath SC, Millay DP. Myoblast fusion confusion: the resolution begins. Skelet Muscle. 2018;8(1):3. https://doi.org/10.1186/s13395017-0149-3

11. Brukman NG, Uygur B, Podbilewicz B, Chernomordik LV. How cells fuse. J Cell Biol. 2019;218(5):1436-51. https://doi.org/10.1083/jcb.201901017.

12. Goenka S, Kaplan MH. Transcriptional regulation by STAT6. Immunol Res. 2011:50(1):87-96. https://doi.org/10.1007/s12026-011-8205-2.

13. Takeda K, Tanaka T, Shi W, Matsumoto M, Minami M, Kashiwamura S, et al. Essential role of Stat6 in IL-4 signalling. Nature. 1996;380(6575):627-30. https://doi.org/10.1038/380627a0

14. Kaplan MH, Daniel C, Schindler U, Grusby MJ. Stat proteins control lymphocyte proliferation by regulating p27Kip1 expression. Mol Cell Biol. 1998;18(4):1996-2003. https://doi.org/10.1128/MCB.18.4.1996.

15. Wurster AL, Withers DJ, Uchida T, White MF, Grusby MJ. Stat6 and IRS-2 cooperate in interleukin 4 (IL-4)-induced proliferation and differentiation but are dispensable for IL-4-dependent rescue from apoptosis. Mol Cell Biol. 2002;22(1):117-26. https://doi.org/10.1128/MCB.22.1.117-126.2002.

16. Moreno JL, Mikhailenko I, Tondravi MM, Keegan AD. IL-4 promotes the formation of multinucleated giant cells from macrophage precursors by a STAT6-dependent, homotypic mechanism: contribution of E-cadherin. J Leukoc Biol. 2007;82(6):1542-53. https://doi.org/10.1189/jlb.0107058.

17. Xu J, Chen Z, Yu F, Liu H, Ma C, Xie D, et al. IL-4/STAT6 signaling facilitates innate hematoma resolution and neurological recovery after hemorrhagic stroke in mice. Proc Natl Acad Sci U S A. 2020;117(51):32679-90. https://doi. org/10.1073/pnas.2018497117.

18. Pavlath GK, Horsley V. Cell fusion in skeletal muscle--central role of NFATC2 in regulating muscle cell size. Cell cycle. 2003;2(5):420-3.

19. Horsley V, Friday BB, Matteson S, Kegley KM, Gephart J, Pavlath GK. Regulation of the growth of multinucleated muscle cells by an NFATC2-dependent pathway. J Cell Biol. 2001;153(2):329-38. https://doi.org/10.1083/jcb.153.2.329.

20. Abbott KL, Friday BB, Thaloor D, Murphy TJ, Pavlath GK. Activation and cellular localization of the cyclosporine A-sensitive transcription factor NF-AT in skeletal muscle cells. Mol Biol Cell. 1998;9(10):2905-16. https://doi.org/10.1 091/mbc.9.10.2905.

21. Horsley V, Jansen KM, Mills ST, Pavlath GK. IL-4 acts as a myoblast recruitment factor during mammalian muscle growth. Cell. 2003;113(4):48394. https://doi.org/10.1016/S0092-8674(03)00319-2.

22. Imayoshi I, Hirano K, Sakamoto M, Miyoshi G, Imura T, Kitano S, et al. A multifunctional teal-fluorescent Rosa26 reporter mouse line for Cre- and Flp-mediated recombination. Neurosci Res. 2012;73(1):85-91. https://doi. org/10.1016/j.neures.2012.02.003.

23. Murphy MM, Lawson JA, Mathew SJ, Hutcheson DA, Kardon G. Satellite cells, connective tissue fibroblasts and their interactions are crucial for muscle regeneration. Development. 2011;138(17):3625-37. https://doi.org/1 $0.1242 /$ dev.064162

24. Kurosaka M, Ogura Y, Funabashi T, Akema T. Involvement of transient receptor potential cation channel vanilloid 1 (TRPV1) in myoblast fusion. J Cell Physiol. 2016;231(10):2275-85. https://doi.org/10.1002/jcp.25345.

25. Ogura $Y$, Hindi SM, Sato S, Xiong G, Akira S, Kumar A. TAK1 modulates satellite stem cell homeostasis and skeletal muscle repair. Nat Commun. 2015;6(1):10123. https://doi.org/10.1038/ncomms10123.

26. Kurosaka M, Ogura Y, Funabashi T, Akema T. Early growth response 3 (Egr3) contributes a maintenance of C2C12 myoblast proliferation. J Cell Physiol. 2017;232(5):1114-22. https://doi.org/10.1002/jcp.25574

27. Schindelin J, Arganda-Carreras I, Frise E, Kaynig V, Longair M, Pietzsch T, et al. Fiji: an open-source platform for biological-image analysis. Nat Methods. 2012;9(7):676-82. https://doi.org/10.1038/nmeth.2019.

28. Millay DP, Sutherland LB, Bassel-Duby R, Olson EN. Myomaker is essential for muscle regeneration. Genes Dev. 2014;28(15):1641-6. https://doi.org/10.11 01/gad.247205.114.

29. Goh Q, Song T, Petrany MJ, Cramer AA, Sun C, Sadayappan S, et al. Myonuclear accretion is a determinant of exercise-induced remodeling in skeletal muscle. Elife. 2019;8. https://doi.org/10.7554/eLife.44876. 
30. Lafuste P, Sonnet C, Chazaud B, Dreyfus PA, Gherardi RK, Wewer UM, et al. ADAM12 and alpha9beta1 integrin are instrumental in human myogenic cell differentiation. Mol Biol Cell. 2005;16(2):861-70. https://doi.org/10.1091/ mbc.e04-03-0226.

31. Madaro L, Marrocco V, Fiore P, Aulino P, Smeriglio P, Adamo S, et al. PKCtheta signaling is required for myoblast fusion by regulating the expression of caveolin-3 and beta1D integrin upstream focal adhesion kinase. Mol Biol Cell. 2011;22(8):1409-19. https://doi.org/10.1091/mbc.e10-1 0-0821.

32. Schwander M, Leu M, Stumm M, Dorchies OM, Ruegg UT, Schittny J, et al. Beta1 integrins regulate myoblast fusion and sarcomere assembly. Dev Cell. 2003;4(5):673-85. https://doi.org/10.1016/S1534-5807(03)00118-7.

33. Cifuentes-Diaz C, Nicolet M, Alameddine H, Goudou D, Dehaupas M, Rieger $F$, et al. M-cadherin localization in developing adult and regenerating mouse skeletal muscle: possible involvement in secondary myogenesis. Mech Dev. 1995;50(1):85-97. https://doi.org/10.1016/0925-4773(94)00327-J.

34. Charrasse S, Comunale F, Grumbach Y, Poulat F, Blangy A, Gauthier-Rouviere C. RhoA GTPase regulates M-cadherin activity and myoblast fusion. Mol Biol Cell. 2006:17(2):749-59. https://doi.org/10.1091/mbc.e05-04-0284.

35. Quach NL, Biressi S, Reichardt LF, Keller C, Rando TA. Focal adhesion kinase signaling regulates the expression of caveolin 3 and beta1 integrin, genes essential for normal myoblast fusion. Mol Biol Cell. 2009;20(14):3422-35. https://doi.org/10.1091/mbc.e09-02-0175.

36. Doherty KR, Cave A, Davis DB, Delmonte AJ, Posey A, Earley JU, et al. Normal myoblast fusion requires myoferlin. Development. 2005;132(24): 5565-75. https://doi.org/10.1242/dev.02155.

37. Lluis F, Ballestar E, Suelves M, Esteller M, Munoz-Canoves P. E47 phosphorylation by p38 MAPK promotes MyoD/E47 association and muscle-specific gene transcription. EMBO J. 2005;24(5):974-84. https://doi. org/10.1038/sj.emboj.7600528.

38. Costamagna D, Duelen R, Penna F, Neumann D, Costelli P, Sampaolesi M. Interleukin-4 administration improves muscle function, adult myogenesis, and lifespan of colon carcinoma-bearing mice. J Cachexia Sarcopenia Muscle. 2020;11(3):783-801. https://doi.org/10.1002/jcsm.12539.

39. Chi $\mathrm{H}$. Regulation and function of mTOR signalling in $\mathrm{T}$ cell fate decisions. Nat Rev Immunol. 2012;12(5):325-38. https://doi.org/10.1038/nri3198.

40. Ge Y, Chen J. Mammalian target of rapamycin (mTOR) signaling network in skeletal myogenesis. J Biol Chem. 2012;287(52):43928-35. https://doi.org/1 0.1074/jbc.R112.406942.

41. Kikani CK, Wu X, Fogarty S, Kang SAW, Dephoure N, Gygi SP, et al. Activation of PASK by $\mathrm{mTORC} 1$ is required for the onset of the terminal differentiation program. Proc Natl Acad Sci U S A. 2019;116(21):10382-91. https://doi.org/10.1073/pnas.1804013116.

42. Wan L, Lin CW, Lin YJ, Sheu JJ, Chen BH, Liao CC, et al. Type I IFN induced IL1-Ra expression in hepatocytes is mediated by activating STAT6 through the formation of STAT2: STAT6 heterodimer. J Cell Mol Med. 2008;12(3):87688. https://doi.org/10.1111/j.1582-4934.2008.00143.x.

43. Franzi S, Salajegheh M, Nazareno R, Greenberg SA. Type 1 interferons inhibit myotube formation independently of upregulation of interferon-stimulated gene 15. PLoS One. 2013;8(6):e65362. https://doi.org/10.1371/journal.pone. 0065362.

44. Gupta S, Jiang M, Pernis AB. IFN-alpha activates Stat6 and leads to the formation of Stat2:Stat6 complexes in B cells. J Immunol. 1999;163(7):383441.

45. Cao H, Zhang J, Liu H, Wan L, Zhang H, Huang Q, et al. IL-13/STAT6 signaling plays a critical role in the epithelial-mesenchymal transition of colorectal cancer cells. Oncotarget. 2016;7(38):61183-98. https://doi.org/10.1 8632/oncotarget.11282

46. Zhou Y, Yu X, Chen H, Sjoberg S, Roux J, Zhang L, et al. Leptin deficiency shifts mast cells toward anti-inflammatory actions and protects mice from obesity and diabetes by polarizing M2 macrophages. Cell Metab. 2015;22(6): 1045-58. https://doi.org/10.1016/j.cmet.2015.09.013.

47. Zetser A, Gredinger E, Bengal E. p38 mitogen-activated protein kinase pathway promotes skeletal muscle differentiation. Participation of the Mef2c transcription factor. J Biol Chem. 1999;274(8):5193-200. https://doi.org/10.1 074/jbc.274.8.5193.

48. Jones NC, Tyner KJ, Nibarger L, Stanley HM, Cornelison DD, Fedorov YV, et al. The p38alpha/beta MAPK functions as a molecular switch to activate the quiescent satellite cell. J Cell Biol. 2005;169(1):105-16. https://doi.org/1 $0.1083 / j \mathrm{cb} .200408066$.
49. Suelves M, Lluis F, Ruiz V, Nebreda AR, Munoz-Canoves P. Phosphorylation of MRF4 transactivation domain by p38 mediates repression of specific myogenic genes. EMBO J. 2004;23(2):365-75. https://doi.org/10.1038/sj. emboj.7600056.

50. Page JL, Wang X, Sordillo LM, Johnson SE. MEKK1 signaling through p38 leads to transcriptional inactivation of E47 and repression of skeletal myogenesis. J Biol Chem. 2004;279(30):30966-72. https://doi.org/10.1074/ jbc.M402224200.

51. Weston AD, Sampaio AV, Ridgeway AG, Underhill TM. Inhibition of p38 MAPK signaling promotes late stages of myogenesis. J Cell Sci. 2003;116(Pt 14):2885-93. https://doi.org/10.1242/jcs.00525.

52. Relaix F, Zammit PS. Satellite cells are essential for skeletal muscle regeneration: the cell on the edge returns centre stage. Development. 2012 139(16):2845-56. https://doi.org/10.1242/dev.069088.

53. Wang J, Conboy I. Embryonic vs. adult myogenesis: challenging the 'regeneration recapitulates development' paradigm. J Mol Cell Biol. 2010; 2(1):1-4. https://doi.org/10.1093/jmcb/mjp027.

54. Bentzinger CF, Wang YX, Rudnicki MA. Building muscle: molecular regulation of myogenesis. Cold Spring Harb Perspect Biol. 2012;4(2):a008342.

55. Chal J, Pourquie O. Making muscle: skeletal myogenesis in vivo and in vitro. Development. 2017;144(12):2104-22. https://doi.org/10.1242/dev.151035.

\section{Publisher's Note}

Springer Nature remains neutral with regard to jurisdictional claims in published maps and institutional affiliations.
Ready to submit your research? Choose BMC and benefit from:

- fast, convenient online submission

- thorough peer review by experienced researchers in your field

- rapid publication on acceptance

- support for research data, including large and complex data types

- gold Open Access which fosters wider collaboration and increased citations

- maximum visibility for your research: over $100 \mathrm{M}$ website views per year

At BMC, research is always in progress.

Learn more biomedcentral.com/submissions 